LIMA, R. P.; SILVA, A. R.; OLIVEIRA, D. M. S. Análise de trilha de atributos físicos na resistência à penetração de um latossolo amarelo. Revista de Agricultura Neotropical, Cassilândia-MS, v. 1, n. 1, p. 65-74, jul./set. 2014.

\title{
ANÁLISE DE TRILHA DE ATRIBUTOS FÍSICOS NA RESISTÊNCIA À PENETRAÇÃO DE UM LATOSSOLO AMARELO
}

\section{RENATO PAIVA DE LIMA ${ }^{1}$, ANDERSON RODRIGO DA SILVA², DENER MÁRCIO DA SILVA OLIVEIRA ${ }^{1}$}

\footnotetext{
${ }^{1}$ Escola Superior de Agricultura "Luiz de Queiroz”, ESALQ/USP, Piracicaba-SP, renato_agro_@hotmail.com, denermsoliveira@gmail.com. ${ }^{2}$ Instituto Federal Goiano, IF-GO, Urutaí, GO, ar.silva@usp.br
}

\begin{abstract}
RESUMO: O estudo das relações entre os atributos físicos do solo é mecanismo importante no monitoramento da compactação. O objetivo do trabalho foi estudar o desdobramento das correlações em efeitos diretos e indiretos, por meio da análise de trilha, de atributos físicos sobre a resistência à penetração de um Latossolo Amarelo. O trabalho foi realizado na Fazenda Experimental Chã-de-Jardim, Areia, PB. As amostras, total de 24, foram coletadas na camada de 0,0-0,2 m, em uma parcela experimental de $35 \times 35 \mathrm{~m}$. Os atributos físicos analisados foram: densidade do solo, umidade, textura, além da resistência à penetração. As variáveis foram submetidas à análise de trilha, considerando a resistência à penetração como variável básica e a densidade do solo, umidade e silte+argila como variáveis explicativas. A densidade do solo foi o único atributo que se correlacionou diretamente com a resistência à penetração, com efeitos bem superiores aos demais atributos físicos do solo analisados. A umidade do solo e as frações texturais não apresentaram correlações significativas com a resistência à penetração de forma direta ou indireta. A densidade do solo foi o atributo que mais influenciou a resistência a penetração de forma direta. $\mathrm{O}$ efeito da umidade e da textura na resistência à penetração é indiretamente influenciado pela densidade do solo.
\end{abstract}

PALAVRAS-CHAVE: compactação do solo, densidade do solo, textura do solo

\section{PHYSICAL ATTRIBUTES PATH ANALYSIS OF PENETRATION RESISTANCE IN A YELLOW LATOSOL}

\begin{abstract}
The study of the relationship among soil physical properties is a crucial mechanism for soil compaction monitoring. The objective of this work was to study correlations decomposition in direct and indirect effects of physical attributes on penetration resistance in a Yellow Latosol using path analysis. The study was conducted in a Yellow Latosol, in the Experimental Farm Chã-de-Jardim, Areia, Paraiba state. The samples, 24 in total, were collected at $0.0-0.2 \mathrm{~m}$, in an experimental plot of $35 \times 35 \mathrm{~m}$. The analyzed physical attributes were: bulk density, soil moisture, texture, beyond the penetration resistance. The variables were subjected to path analysis, considering the penetration resistance as basic variable and bulk density, soil moisture and silt + clay as explanatory variables. Soil bulk density was the single attribute that correlated directly with the penetration resistance, with superior effects to other analyzed soil physical properties. Soil moisture and textural fractions showed no significant correlations with directly or indirectly penetration resistance. Soil bulk density was the attribute that most influenced the directly penetration resistance. The moisture and texture effects on penetration resistance are indirectly influenced by soil density.
\end{abstract}

KEY-WORDS: soil compaction, bulk density, soil texture 


\section{INTRODUÇÃO}

A compactação do solo pelo uso de práticas inadequadas de manejo resultam diretamente em aumento na densidade do solo, diminuição do teor de umidade e aumento da resistência à penetração (AMPOORTER et al., 2010; HAN et al., 2011), sendo um fator altamente influenciado pela textura (RIBON; TAVARES FILHO, 2008). Segundo Dalchiavon et al. (2011), em consequência destas alterações sofridas nos atributos físicos do solo e formação de camadas compactadas, pode haver redução na penetração das raízes no solo, alterando o equilíbrio na proporção de gases do solo e a disponibilidade de água e nutrientes às plantas.

Devido às relações encontradas entre os componentes físicos do solo, o estudo associativo e conhecimento dos fatores de causa e efeito entre estes atributos são de fundamental importância para escolha do sistema de preparo mais adequado ao crescimento e desenvolvimento das culturas. A compactação, que envolve relações entre diversos atributos estruturais do solo, é influenciada, principalmente, pela umidade do solo (HAN et al., 2011, GAO et al., 2012) e textura (RIBON; TAVARES FILHO, 2008; AMPOORTER et al., 2010), sendo a densidade do solo e a resistência à penetração atributos diagnósticos desse movimento mecânico (FREITAS et al., 2011; GAO et al., 2012).

A resistência à penetração é um dos principais indicadores para o diagnóstico da compactação e há um esforço para entender quais os atributos físicos do solo que mais influenciam a variação da resistência a penetração no campo (VAZ et al., 2011). Algumas relações já estão estabelecidas na literatura (ROBOREDO et al., 2010; SILVEIRA et al., 2010), tais como as correlações positivas e negativas encontradas frequentemente para densidade e umidade do solo, respectivamente. No entanto, as correlações encontradas para estes atributos são realizadas entre pares de variáveis, ou seja, a correlação de uma variável com a outra, apontando um coeficiente linear direto entre as mesmas.

O coeficiente de correlação linear simples indica que há relação linear entre duas variáveis, e a mudança de uma implica mudança constante no valor médio de outra variável, positiva ou negativamente. No entanto, uma vez que na ciência do solo os fatores ambientais relacionam-se de maneira interativa e complexa, o estudo da correlação simples torna-se insuficiente (PACHECO; CANTALICE, 2011), e pode está sendo utilizada incorretamente na identificação de correlações para construção de modelos de predição ou funções de pedotransferências, por exemplo.

Uma técnica bastante eficiente para o estudo do efeito de uma variável em outra, é análise trilha, que fornece valores, denominados de coeficiente de trilha, que medem a relação linear direta de uma variável sobre a outra, no contexto de causa e efeito (PACHECO; CANTALICE, 2011; SILVA et al., 2013) pelo desdobramento das correlações. Esta estratégia permite conhecer qual a contribuição que outras variáveis exerceram dentro do efeito total (correlação) de uma variável explicativa a resposta, quando estas são analisadas conjuntamente (SILVA et al., 2013).

Sabendo que em física do solo, por se tratar de uma esfera da ciência do solo que envolve muitas relações estruturais, que dependem umas das outras, o conhecimento das relações entre propriedades físicas e mecânicas do solo pode contribuir no desenvolvimento de funções de pedotransferência (IMHOFF et al., 2004), que permitam estimar outras propriedades do solo de difícil mensuração (SUZUKI et al., 2008). No entanto, é necessário se conhecer ao certo à proporção que um determinado atributo exerce sobre o outro.

Dentro deste contexto, nos estudos de física do solo, a resistência à penetração é considerada uma variável indicadora de compactação e resistência ao crescimento de raízes, 
podendo ser utilizada como diagnóstico de degradação, sendo uma variável altamente dependente da textura, umidade e densidade, o que justifica os estudos detalhados na variação deste atributo no campo (PACHECO; CANTALICE, 2011).

O objetivo deste trabalho foi estudar, por meio da análise de trilha, o desdobramento das correlações em efeitos diretos e indiretos de atributos físicos do solo sobre a resistência à penetração em um Latossolo Amarelo.

\section{MATERIAL E MÉTODOS}

O trabalho foi realizado no período de agosto de 2010 a maio de 2011, na Fazenda Experimental Chã-de-Jardim, Centro de Ciências Agrárias da Universidade Federal da Paraíba, município de Areia (Figura 1), localizado na microrregião do Brejo Paraibano, sob as coordenadas geográficas $6^{\circ} 58^{\prime} 12^{\prime \prime} \mathrm{S}$ e $35^{\circ} 42^{\prime} 15^{\prime \prime} \mathrm{W}$. Segundo a classificação de Köppen, o clima é As', quente e úmido com chuvas de outono-inverno com precipitações médias anuais de 1.200 a $1.400 \mathrm{~mm}$ (sendo que mais de $75 \%$ destas estão concentradas no período de março a agosto), temperatura média anual oscilando entre 22 e $26^{\circ} \mathrm{C}$ e umidade relativa do ar entre 75 e $87 \%$.

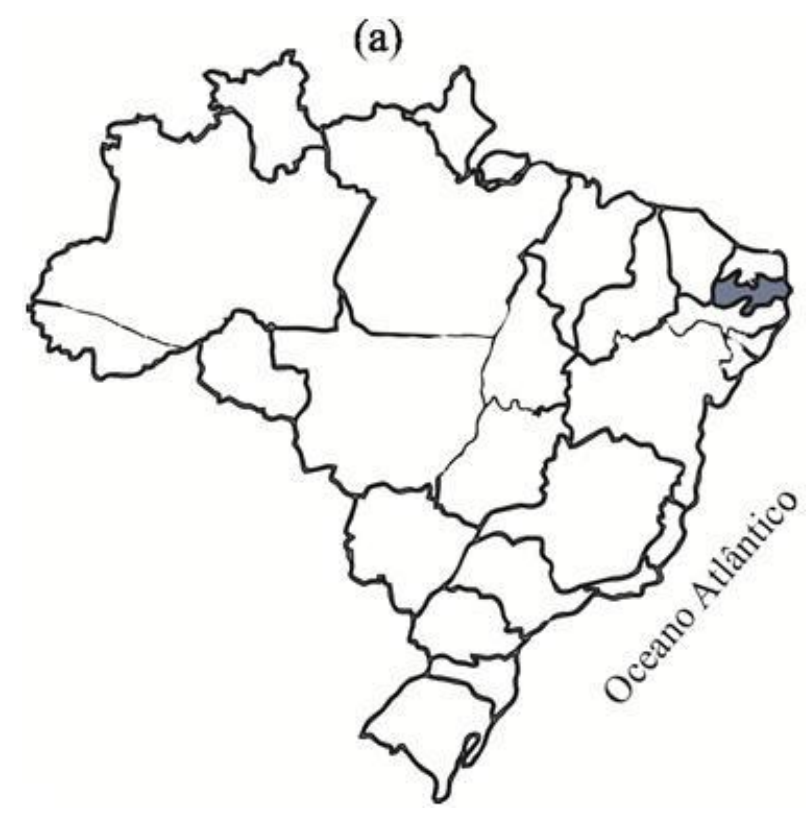

(b)

Figura 1. Mapa do município de Areia (b), Paraíba, Brasil (a), local do experimento.

A área em estudo vinha sendo utilizada sob intervalos de cultivos nos últimos 25 anos e no momento da amostragem estava sob pousio após colheita de milho. No último cultivo, no caso o milho, o preparo do solo foi realizado de forma convencional, com uma aração e uma gradagem, na profundidade de 0,0-0,2 m. A semeadura foi realizada manualmente e não houve adubação na cultura.

O estudo foi conduzido em área experimental de Latossolo Amarelo (EMBRAPA, 2006), cuja granulometria e a classificação textural se encontram na Tabela 1. As amostras, em número total de 24 , foram coletadas na camada de $0,0-0,2 \mathrm{~m}$, em uma parcela de $35 \times 35$ $\mathrm{m}$, instalada exclusivamente para coleta dos dados, amostrados de forma aleatória dentro da parcela. 
Tabela 1. Granulometria e classificação textural da área experimental, Fazenda Chã-doJardim, Areia, PB.

\begin{tabular}{ccc}
\hline Areia & Silte & Argila \\
& & \\
537 & g kg & 386 \\
\hline
\end{tabular}

Os atributos físicos analisados foram: densidade do solo (DS) $\left(\mathrm{Mg} \mathrm{m}^{-3}\right)$, umidade gravimétrica (US) $\left(\mathrm{kg} \mathrm{kg}^{-1}\right)$, textura $\left(\mathrm{g} \mathrm{kg}^{-1}\right)$, além da resistência mecânica à penetração (RP) $(\mathrm{MPa})$. Anéis metálicos de volume conhecido $\left(90,5 \mathrm{~cm}^{3}\right)$ foram utilizados na coleta de amostras não deformadas para determinação da densidade do solo. As amostras foram coletadas e transportadas até o Laboratório de Física do Solo do Centro de Ciências Agrárias da Universidade Federal da Paraíba, onde foi determinado o teor de umidade através do método padrão da estufa e realizadas as análises texturais pelo método do densímetro, conforme metodologia da EMBRAPA (1997). Para coleta dos dados de resistência mecânica à penetração foi utilizado um penetrômetro de impacto modelo IAA/Planalsucar, com metodologia sugerida por Stolf (1991).

Na Figura 2 apresenta-se um diagrama causal previamente estabelecido, considerandose a resistência à penetração (RP) como variável básica; a seta unidirecional indica efeito direto de cada variável explicativa sobre a variável básica; a seta bidirecional representa a interdependência entre duas variáveis explicativas, cuja magnitude é quantificada pela correlação.

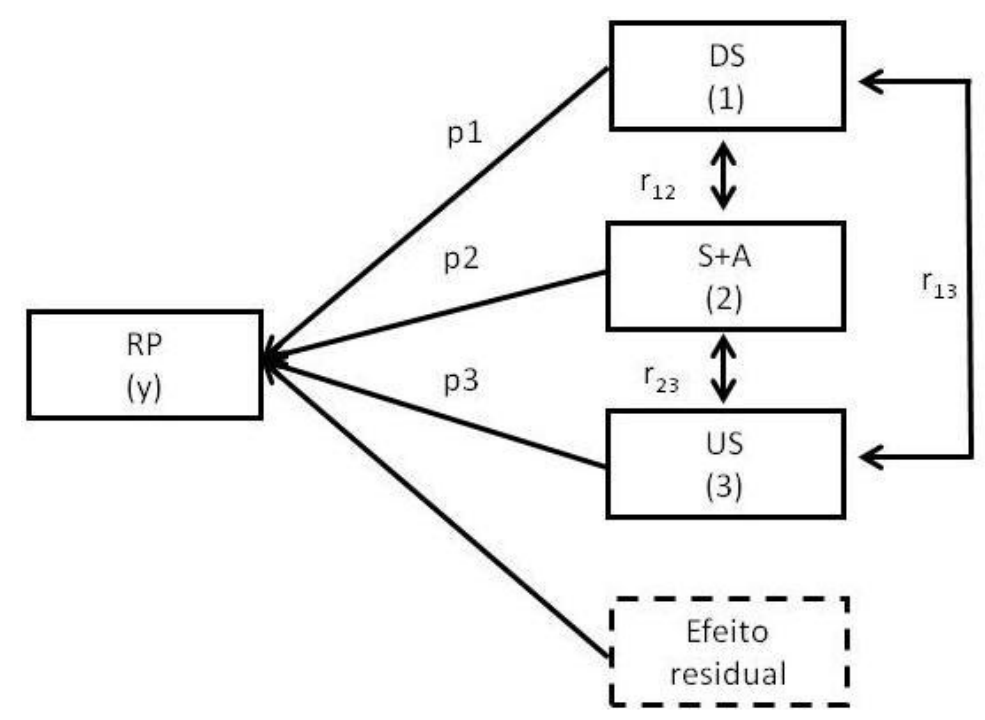

Figura 2. Diagrama causal com indicação dos efeitos diretos (p) das variáveis explicativas sobre a variável básica resistência à penetração (RP), e correlação (r) entre variáveis explicativas: densidade do solo DS (1), quantidade de silte+argila (S+A) (2) e umidade do solo (US) (3). 
Portanto, para a análise de trilha, foram consideradas a RP com variável dependente e a DS, (silte+argila) S+A (PACHECO; CANTALICE, 2011) e US, como variáveis explicativas.

Antes de proceder à estimação dos coeficientes de trilha, foi realizado diagnóstico de multicolinearidade da matriz de correlações entre as variáveis explicativas segundo critério proposto por Montgomery e Peck (1981), que é baseado no número de condição (NC), que consiste na razão entre o maior e o menor autovalor da matriz de correlações, em que: $\mathrm{NC}<$ 100 - multicolinearidade fraca; $100<\mathrm{NC}<1000$ - multicolinearidade moderada a forte; NC $>1000$ - multicolinearidade severa. Ademais, Montgomery e Runger (2008) relatam que a presença de multicolinearidade também pode ser facilmente verificada pela magnitude dos fatores de inflação da variância (FIV), que são os elementos diagonais da inversa da matriz de correlações, de modo que se qualquer VIF exceder 10, então a multicolinearidade será um problema.

As análises de trilha dos atributos físicos do solo sobre a resistência à penetração, bem como o diagnóstico de multicolinearidade, foram realizados utilizando a função pathanalysis() do pacote biotools versão 1.1 (SILVA, 2014), software R versão 3.0.2 (R CORE TEAM, 2014).

\section{RESULTADOS E DISCUSSÃO}

A análise estatística descritiva para a DS, S+A, US e RP são apresentados na Tabela 2. Segundo a classificação de Warrick e Nielsen (1980), que considera o coeficiente de variação baixo $(\mathrm{CV}<12 \%)$, média $(12<\mathrm{CV}<62 \%)$ e alta $(\mathrm{CV}>62 \%)$, apenas os valores de RP apresentaram variação média, os demais apresentam variação baixa, corroborando com os obtidos por diversos autores (CAVALCANTE et al., 2011; SANTOS et al., 2012) quando analisaram o coeficiente de variação de atributos físicos do solo.

Tabela 2. Análise estatística descritiva $(n=24)$ para DS, S+A, US e RP em Latossolo Amarelo.

\begin{tabular}{lcccc}
\hline \multirow{2}{*}{ Variável descritiva } & $\mathrm{DS}$ & $\mathrm{S}+\mathrm{A}$ & $\mathrm{US}$ & $\mathrm{RP}$ \\
\cline { 2 - 5 } & $\mathrm{Mg} \mathrm{m}^{-3}$ & $\mathrm{~g} \mathrm{~kg}^{-1}$ & $\mathrm{~kg} \mathrm{~kg}^{-1}$ & $\mathrm{MPa}$ \\
\hline Média & 1,31 & 463,12 & 24,36 & 1,75 \\
Desvio padrão & 0,09 & 26,68 & 2,61 & 0,25 \\
Mínimo & 1,12 & 411,62 & 19,80 & 1,32 \\
Máximo & 1,43 & 522,73 & 29,64 & 2,28 \\
\hline CV $(\%)$ & 6,60 & 5,76 & 10,72 & 14,46 \\
Classificação $(\mathrm{CV})$ & Baixa & Baixa & Baixa & Média \\
\hline
\end{tabular}

$\overline{\mathrm{CV}}$ - coeficiente de variação (\%)

Os valores do CV para os atributos DS, S+A e US foram considerados baixos de acordo com a classificação de Warrick e Nielsen (1980), não ultrapassando $10 \%$. Santos et al. (2012) verificaram valores semelhantes para DS quando estudaram variabilidade espacial de atributos físicos do solo e afirmam que para este atributo não são comuns valores superiores a $10 \%$ de CV, mesmo com as variações importas pelo manejo. Cavalcante et al. (2011) em trabalho sobre variabilidade espacial de atributos físicos do solo sob diferentes usos e manejos, verificaram valores semelhantes aos da Tabela 2 para CV da US. No entanto, em ambos os trabalhos (SANTOS et al., 2012; CAVALCANTE et al., 2011) foram verificados 
valores de CV próximos a 50\% para RP e a $20 \%$ para silte e argila, discordando dos valores da Tabela 2 para estes atributos.

Os valores elevados de CV para RP podem ser observados em diversos trabalhos (CHAVES et al., 2012; CAVALCANTE et al., 2011), e o fato deste atributo apresentar alta variação pode estar associado à dependência que seus valores apresentam dos demais atributos físicos do solo. Variações na DS e US em determinado sistema de manejo, principalmente aqueles que sofrem influência do tráfego, podem exercer grandes variações na RP. O fato do S+A e a RP apresentarem valores inferiores ao encontrado em outros trabalhos pode estar relacionado à condição de revolvimento do solo da camada em estudo na ocasião do preparo solo, que pode ter homogeneizado a área experimental culminando na quebra da estrutura do solo, refletindo em baixos $\mathrm{CV}$ para todos os atributos analisados.

Altos CV para RP, superiores aos da Tabela 2, podem ser observados nos trabalhos realizados por Chaves et al. (2012) e Cavalcante et al. (2011), que estudaram a variação dos atributos físicos em diversos sistemas de manejo. No trabalho de Chaves et al. (2012), por exemplo, pode-se verificar CV dez vezes maior para RP em relação a DS. Portanto, diferente dos sistemas de manejo que mantém a estrutura do solo preservada, o revolvimento por ocasião do preparo foi determinante para que a área experimental apresentasse homogeneidade com relação aos atributos físicos do solo.

Com relação aos valores máximos de RP obtidos na área experimental, verificou-se que foram próximos a 2,20 $\mathrm{MPa}(2,28 \mathrm{MPa})$. Embora não haja consenso na literatura sobre um limite crítico de RP que seja universalmente restritivo ao desenvolvimento radicular, por este atributo ser dependente da textura do solo, o valor de RP = 2,0 MPa (BLAINSKI et al., 2008; TORMENA et al., 2002) tem sido frequentemente utilizado como crítico para o crescimento das plantas. Com base nos valores máximos de RP da Tabela 2, pode-se verificar que a área apresenta pontos que podem restringir o desenvolvimento radicular das plantas.

Na Tabela 3 é apresentado o resultado da análise de trilha da RP em função da DS, S+A e US, em que o diagnóstico de multicolinearidade revelou número de condição (NC) de 3,10, o que não constitui problema para a análise de trilha, conforme critério de Montgomery e Peck (1981), que consideram multicolinearidade fraca para valores de NC menores que 100. Além disso, os valores dos fatores de inflação da variância (FIV) foram de 1,35, 1,19 e 1,21, para DS, S+A e US, respectivamente, menores que 10, conforme critérios de Montgomery e Runger (2008). Portanto, o efeito adverso da multicolinearidade está contornado, o que permitiu a interpretação adequada dos coeficientes de trilha. Segundo Coimbra et al. (2005), quando o efeito adverso da multicolinearidade não é superado, pode causar uma superestimativa dos efeitos diretos das variáveis explicativas sobre a variável resposta, comprometendo a interpretação dos resultados.

Os coeficientes de trilha dos atributos físicos (DS, S+A e US) estudados sobre a RP são apresentados na Tabela 3. O modelo de análise de trilha adotado explicou $91 \%\left(\mathrm{R}^{2}\right)$ das variações na RP. As variáveis, em ordem decrescente, que apresentaram maior correlação com a variável básica (RP) foram: DS, S+A, e US. Observa-se que a DS apresentou maior efeito direto $(0,89)$ sobre a RP, com coeficiente positivo, indicando que o aumento da densidade contribuiu para a elevação da RP. Já os efeitos diretos da US $(0,05)$ e S+A $(0,11)$ não apresentaram efeitos significativos na RP, fato observado pelos valores de seus coeficientes, que ficaram abaixo do efeito da variável residual $(0,28)$, eliminando a possibilidade de uma análise e interpretação dos efeitos desses atributos sobre a RP de forma direta. 
Tabela 3. Análise de trilha da variável básica (RP) sob as estimativas dos efeitos diretos (diagonal) e indiretos das variáveis explicativas: DS, S+A e US.

\begin{tabular}{ccccc}
\hline Atributo & DS & S+A & US & Efeito Total \\
\hline DS & 0,89 & 0,03 & 0,01 & 0,94 \\
S+A & 0,29 & 0,11 & $-0,01$ & 0,40 \\
US & 0,31 & $-0,01$ & 0,05 & 0,35 \\
\hline
\end{tabular}

Coeficiente de determinação $\left(\mathrm{R}^{2}\right)=0,91$

Efeito da variável residual $\left(\mathrm{p}_{\mathrm{E}}\right)=0,28$

FIV

$\begin{array}{ccc}\text {------- Diagnóstico de Multicolinearidade -- } \\ 1,35 & 1,19\end{array}$

$\mathrm{NC}=3,10$

Diagnóstico: fraca

FIV - fator de inflação da variância; NC - número de condição.

Vários trabalhos (SILVEIRA et al., 2010; VAZ et al., 2011) demonstraram relação da RP com a US de forma inversamente proporcional, ou seja, um aumento da RP do solo estaria associado necessariamente a uma queda nos valores de US. No entanto, alguns desses trabalhos apresentam uma relação não linear entres estas variáveis (VAZ et al., 2011), o que não é considerado na análise de trilha, já que se trata de uma análise de relações lineares. Estas relações não lineares entre a RP e US podem ser observadas nos modelos apresentados por Busscher (1990) e Silveira et al. (2010).

O efeito direto dos conteúdos de S+A foi baixo, inferior a variável residual. Embora a literatura mencione o efeito da textura na RP, as diferenças granulométricas parecem exercer influência significativa apenas quando são analisadas em diferentes classes texturais, como mostra os resultados obtidos por Lima et al. (2013), que estudou o efeito de três diferentes classes texturais na RP e observou diferenças significativas dentro destas classes, evidenciando o efeito da textura nos valores de RP. Portanto, as variações impostas pela variabilidade espacial da área experimental (Tabela 2) na textura, não afetou a RP a ponto de poder ser observada no efeito direto do coeficiente de trilha apresentado pelo atributo $\mathrm{S}+\mathrm{A}$.

Os efeitos indiretos das variáveis S+A e US via DS foram baixos $(0,03$ e 0,01 , respectivamente), apresentando valores bem inferiores à variável residual. Já o efeito da DS via $S+A$ e US $(0,29$ e 0,31 , respectivamente) foram ligeiramente maiores que a variável residual. No entanto, bem inferior ao efeito direto da DS, o que demonstra que a DS dominou a correlação com RP.

De acordo com a análise de trilha da Tabela 3, a DS foi o atributo físico do solo que apresentou maior correlação direta com a RP, relação positiva. Roboredo et al. (2010) encontraram correlação positiva $(\mathrm{r}=0,91)$ quando estudaram $\mathrm{RP}$ e densidade do solo, afirmando que a primeira aumenta com a elevação da segunda. A RP está diretamente correlacionada com vários atributos e condições estruturais do solo, dentre eles a densidade (SILVEIRA et al., 2010).

Benedetti et al. (2010) avaliaram RP em diferentes sistemas de uso do solo correlacionando os valores com os de densidade e concluíram que a resistência à penetração foi altamente influenciada pela densidade do solo. Altos valores de densidades do solo reduzem os espaços vazios, aumentando a resistência mecânica dos solos e, em consequência, prejudicam o desenvolvimento do sistema radicular das culturas (RIBON et al., 2008).

Em trabalho especifico com a técnica da análise de trilha em atributos físicos do solo e matéria orgânica sobre a RP, Pacheco e Cantalice (2011) concluíram que a DS foi o atributo 
mais importante e também o único que apresentou coeficiente de trilha com valor superior ao do efeito da variável residual, confirmando seu efeito direto, com mesmo sinal, sobre RP, concordando com os resultados encontrados neste trabalho. Portanto, os solos mais densos tendem a apresentar maiores valores de RP, e nestas condições experimentais, sem qualquer ou mínima influência de outros atributos físicos do solo.

\section{CONCLUSÕES}

A densidade do solo foi o atributo que mais influenciou na resistência à penetração de forma direta.

O efeito da umidade e da textura na resistência à penetração é indiretamente influenciado pela densidade do solo.

\section{REFERENCIAS BIBLIOGRÁFICAS}

AMPOORTER, E.; VAN NEVEL, L.; DE VOS, B.; HERMY, M.; VERHEYEN, K. Assessing the effects of initial soil characteristics, machine mass and traffic intensity on forest soil compaction. Forest Ecology and Management, v. 260, n. 10, p. 1664-1676, 2010.

BENEDETTI, M. M.; DUARTE, I. N.; MELO JUNIOR, H. B.; BORGES, E. N. Resistência do solo à penetração em um Latossolo Vermelho distrófico típico sob diferentes usos. Enciclopédia Biosfera, Goiânia-GO, v. 6, n. 11, p. 1-9, 2010.

BLAINSKI, E.; TORMENA, C. A.; FIDALSKI, J.; GUIMARÃES, R. M. L. Quantificação da degradação física do solo por meio da curva de resistência do solo à penetração. Revista Brasileira de Ciência do Solo, Viçosa-MG, v. 32, n. 3, p. 975-983, 2008.

BUSSCHER, W. J. Adjustment of flat-tipped penetrometer resistance data to common water content. Transactions of the ASAE, Saint Joseph-Michigan-USA, v. 33, n. 2, p. 519-524, 1990.

CAVAlCAnTE, E. G. S.; ALVES, M. C.; SOUZA, Z. M.; PEREIRA, G. Variabilidade espacial de atributos físicos do solo sob diferentes usos e manejos. Revista Brasileira de Engenharia Agrícola e Ambiental, Campina Grande-PB, v. 15, n. 3, p. 237-243, 2011.

CHAVES, A. A. A.; LACERDA, M. P. C.; GOEDERT, W. J.; RAMOS, M. L. G.; KATO, E. Indicadores de qualidade de Latossolo Vermelho sob diferentes usos. Pesquisa Agropecuária Tropical, Goiânia-GO, v. 42, n. 4, p. 446-454, 2012.

COIMBRA, J.L.M.; BENIN, G.; VIEIRA, E.A.; OLIVEIRA, A.C.; CARVALHO, F.I.F.; GUIDOLIN, A.F.; SOARES, A.P. Consequências da multicolinearidade sobre a análise de trilha em Canola. Ciência Rural, Santa Maria-RS, v. 35, n. 2, p. 347-352, 2005.

DALCHIAVON, F. C.; CARVALHO, M. P..; NOGUEIRA, D. C.; ROMANO, D.; ABRANTES, F. L.; ASSIS, J. T.; OLIVEIRA, M. S. Produtividade da soja e resistência mecânica à penetração do solo sob sistema plantio direto no Cerrado brasileiro. Pesquisa Agropecuária Tropical, Goiânia-GO, v. 41, n. 1, p. 8-19, 2011.

EMBRAPA. EMPRESA BRASILEIRA DE PESQUISA AGROPECUARIA. Manual de métodos de análises de solo. 2. ed. Rio de Janeiro-RJ: Embrapa, 1997. 212 p.

EMBRAPA. EMPRESA BRASILEIRA DE PESQUISA AGROPECUÁRIA. Centro Nacional de Pesquisa em Solos. Sistema Brasileiro de Classificação de Solos. Rio de Janeiro-RJ: Embrapa, 2006. 306 p. 
FREITAS, L.; CASAGRANDE, J. C.; DESUÓ, I. C. Atributos físicos e químicos de solos cultivado com cana-de-açúcar próximo a fragmento nativo. Holos Envolviment, Rio ClaroSP, v. 11, n. 2, p. 137-146, 2011.

GAO, W.; WATTS, C.W.; REN, T.; WHALLEY, W.R. The effects of compaction and soil drying on penetrometer resistance. Soil \& Tillage Research, Craibstone Estate-AberdeenUK, v. 125, p. 14-22, 2012.

HAN, W.; SHI, S.; ZHU, Z.; XUE, L.; JUANLI, Z. A. A simulation test of the impact on soil moisture by agricultural machinery. African Journal of Biotechnology, New Ogorode RoadSapele-Nigeria, v. 10, n. 73, p. 16536-16540, 2011.

IMHOFF, S.; SILVA, A. P.; FALLOW, D. Susceptibility to compaction, load support capacity, and soil compressibility of Hapludox. Soil Science Society of America Journal, Madison-USA v. 68, n. 1, p. 17-24, 2004.

LIMA, R. P.; LEON, M. J.; SILVA, A. R. Compactação do solo de diferentes classes texturais em áreas de produção de cana-de-açúcar. Revista Ceres, Viçosa-MG, v. 60, n. 1, p. 16-20, 2013.

MONTGOMERY, D. C.; PECK, E. A. Introduction to linear regression analysis. New York-USA: John Wiley \& Sons, 1981. 504p.

MONTGOMERY, D. C.; RUNGER, R. C. Estatística aplicada e probabilidade para engenheiros. (Trad. Verônica Calado). Rio de Janeiro-RJ: LTC, 2008. 463 p.

PACHECO, E. P.; CANTALICE, J. R. B. Análise de trilha no estudo dos efeitos de atributos físicos e matéria orgânica sobre a compressibilidade e resistência à penetração de um Argissolo cultivado com cana-de-açúcar. Revista Brasileira de Ciência do Solo, ViçosaMG, v. 35, n. 2, p. 417-428, 2011.

R CORE TEAM. R. A language and environment for statistical computing. Viena, $\mathrm{R}$ Foundation for Statistical Computing, 2013. Disponível em: 〈http://www.R-project.org/>. Acessado em 04 de janeiro de 2014

RIBON, A. A.; TAVARES FILHO, J. Estimativa da resistência mecânica à penetração de um Latossolo vermelho sob cultura perene no Norte do Estado do Paraná. Revista Brasileira de Ciência do Solo, Viçosa-MG, v. 32, n. 5, p. 1817-1825, 2008.

ROBOREDO, D.; MAIA, J. C. S. M.; OLIVEIRA, O. J.; ROQUE, C. G. Uso de dois penetrômetros na avaliação da resistência mecânica de um Latossolo Vermelho distrófico. Engenharia Agrícola, Jaboticabal-SP, v. 30, n. 2, p. 307-314, 2010.

SANTOS, K. S.; MONTENEGRO, A. A. A.; ALMEIDA, B. G.; MONTENEGRO, S. M. G. L, ANDRADE, T. S.; FONTES JUNIOR, R. V. P. Variabilidade espacial de atributos físicos em solos de vale aluvial no semiárido de Pernambuco. Revista Brasileira de Engenharia Agrícola e Ambiental, Campina Grande-PB, v. 16, n. 8, p. 828-835, 2012.

SILVA, A. R. Biotools: Tools for Biometry and Applied Statistics in Agricultural Science. R package version 1.1, 2014. Disponível em: 〈http://CRAN.R-project.org/package=biotools〉. Acessado em 17 fev. 2014.

SILVA, A. R.; NASCIMENTO, M.; CECON, P. R.; SAPUCAY, M. J. C. C.; REGO, E. R.; BARBOSA, L. A. Path analysis in multicollinearity for fruit traits of pepper. Idesia, AricaChile, v.31, n. 2, p.55-60, 2013.

SILVEIRA, D.C.; MELO FILHO, J.F.; SACRAMENTO, J.A.A.S.; SILVEIRA, E.C.P. Relação umidade versus resistência à penetração para um Argissolo Amarelo distrocoeso no 
Recôncavo da Bahia. Revista Brasileira de Ciência do Solo, Viçosa-MG, v. 34, n. 3, p. 659667, 2010.

STOLF, R. Teoria e teste experimental de fórmulas de transformação dos dados de penetrômetro de impacto em resistência do solo. Revista Brasileira de Ciência do Solo, Campinas-SP, v. 15, n. 2, p. 229-35, 1991.

SUZUKI, L. E. A. S.; REINERT, D. J.; REICHERT, J. M.; LIMA, C. L. R. Estimativa da susceptibilidade à compactação e do suporte de carga do solo com base em propriedades físicas de solos do Rio Grande do Sul. Revista Brasileira de Ciência do Solo, Viçosa-MG, v. 32, n. 3, p. 963-973, 2008.

TORMENA, C. A.; BARBOSA, M. C.; COSTA, A. C. S. Densidade, porosidade e resistência a penetração em Latossolo cultivado sob diferentes sistemas de preparo do solo. Scientia Agrícola, Piracicaba-SP, v. 59, n. 4, p. 795-801, 2002.

VAZ, C. M. P.; MANIERI, J. M.; MARIA, I. C.; TULLER, M. Modeling and correction of soil penetration resistance for varying soil water content. Geoderma, v. 166, v. 1, p. 92-101, 2011.

WARRICK, A.W.; NIELSEN, D.R. Spatial variability of soil physical properties in the field. In: HILLEL, D. (Ed.). Application of soil physics. New York: Academic Press, 1980. p. 319344. 